\title{
Genomic profiling identifies common HPV-associated chromosomal alterations in squamous cell carcinomas of cervix and head and neck
}

\author{
Saskia M Wilting ${ }^{\dagger 1}$, Serge J Smeets ${ }^{\dagger 1,2}$, Peter JF Snijders ${ }^{1}$, Wessel N van \\ Wieringen ${ }^{3}$, Mark A van de Wiel ${ }^{3}$, Gerrit A Meijer ${ }^{1}$, Bauke Ylstra1 ${ }^{1}$ C \\ René Leemans ${ }^{2}$, Chris JLM Meijer ${ }^{1}$, Ruud H Brakenhoff ${ }^{2}$, \\ Boudewijn JM Braakhuis ${ }^{\dagger 2}$ and Renske DM Steenbergen*†1
}

\begin{abstract}
Address: ${ }^{1}$ Department of Pathology, VU University Medical Center, Amsterdam, The Netherlands, ${ }^{2}$ Department of Otolaryngology/Head-Neck Surgery, VU University Medical Center, Amsterdam, The Netherlands and ${ }^{3}$ Department of Epidemiology and Biostatistics, VU University Medical Center, Amsterdam, The Netherlands

Email: Saskia M Wilting - s.wilting@vumc.nl; Serge J Smeets - s.smeets@vumc.nl; Peter JF Snijders - pjf.snijders@vumc.nl; Wessel N van Wieringen - wvanwie@few.vu.nl; Mark A van de Wiel - mark.vdwiel@vumc.nl; Gerrit A Meijer - ga.meijer@vumc.nl; Bauke Ylstra - b.ylstra@vumc.nl; C René Leemans - chr.leemans@vumc.nl; Chris JLM Meijer - cjlm.meijer@vumc.nl; Ruud H Brakenhoff - rh.brakenhoff@vumc.nl; Boudewijn JM Braakhuis - bjm.braakhuis@vumc.nl;

Renske DM Steenbergen* - r.steenbergen@vumc.nl

* Corresponding author †Equal contributors
\end{abstract}

Published: I June 2009

BMC Medical Genomics 2009, 2:32 doi:10.1186/1755-8794-2-32
Received: 14 November 2008

Accepted: I June 2009

This article is available from: http://www.biomedcentral.com/1755-8794/2/32

(c) 2009 Wilting et al; licensee BioMed Central Ltd.

This is an Open Access article distributed under the terms of the Creative Commons Attribution License (http://creativecommons.org/licenses/by/2.0), which permits unrestricted use, distribution, and reproduction in any medium, provided the original work is properly cited.

\begin{abstract}
Background: It is well known that a persistent infection with high-risk human papillomavirus (hrHPV) is causally involved in the development of squamous cell carcinomas of the uterine cervix (CxSCCs) and a subset of SCCs of the head and neck (HNSCCs). The latter differ from hrHPVnegative HNSCCs at the clinical and molecular level.
\end{abstract}

Methods: To determine whether hrHPV-associated SCCs arising from different organs have specific chromosomal alterations in common, we compared genome-wide chromosomal profiles of $10 \mathrm{CxSCCs}$ (all hrHPV-positive) with $12 \mathrm{hrHPV}$-positive HNSCCs and $30 \mathrm{hrHPV}$-negative HNSCCs. Potential organ-specific alterations and alterations shared by SCCs in general were investigated as well.

Results: Unsupervised hierarchical clustering resulted in one mainly hrHPV-positive and one mainly hrHPV-negative cluster. Interestingly, loss at $13 q$ and gain at $20 q$ were frequent in HPVpositive carcinomas of both origins, but uncommon in hrHPV-negative HNSCCs, indicating that these alterations are associated with hrHPV-mediated carcinogenesis. Within the group of hrHPVpositive carcinomas, HNSCCs more frequently showed gains of multiple regions at $8 q$ whereas CxSCCs more often showed loss at 17p. Finally, gains at 3q24-29 and losses at I I q22.3-25 were frequent $(>50 \%)$ in all sample groups.

Conclusion: In this study hrHPV-specific, organ-specific, and pan-SCC chromosomal alterations were identified. The existence of hrHPV-specific alterations in SCCs of different anatomical origin, suggests that these alterations are crucial for hrHPV-mediated carcinogenesis. 


\section{Background}

In the pathogenesis of uterine cervical cancer the necessary and causal involvement of high-risk types of the human papillomavirus (hrHPV) is widely accepted and supported by strong epidemiological and molecular evidence [1]. HrHPV is present in virtually all cervical carcinomas and the viral oncogenes E6 and E7 are consistently expressed in cervical cancers and precancers. Deregulated expression of these oncogenes in the basal, dividing cells of the epithelium interferes with cell cycle control due to their ability to induce degradation of the tumour suppressor proteins $\mathrm{p} 53$ and $\mathrm{pRb}$, respectively. This results in uncontrolled cell proliferation and accumulation of specific (epi)genetic changes in the host cell genome, driving progression to a malignant phenotype $[2,3]$. In a previous study we have used array-based comparative genomic hybridisation (array CGH) to determine frequent chromosomal alterations in cervical cancer, which included gains at $1 \mathrm{q}, 3 \mathrm{q}$ and $20 \mathrm{q}$ and losses at $8 \mathrm{q}, 10 \mathrm{q}, 11 \mathrm{q}$, and $13 q$ [4]. The necessity of these and other additional (epi)genetic alterations in the carcinogenic process is illustrated by the fact that their frequency increases with increasing severity of cervical disease.

On the other hand, head and neck squamous cell carcinoma (HNSCC) is known to be mainly caused by wellestablished life-style related habits, such as tobacco and excessive alcohol consumption. However, besides the influence of these life-style carcinogens, high risk human papillomavirus (hrHPV) is present in $15-35 \%$ of HNSCCs and has been suggested to be a separate aetiological factor in head-and-neck carcinogenesis [5-8]. Several studies have shown that hrHPV-positive HNSCCs are associated with a better clinical outcome [7-9]. Moreover, molecular differences were found between hrHPV-positive and hrHPV-negative HNSCCs, supporting the idea of two separate carcinogenic pathways to HNSCC, one determined by life-style carcinogens and the other by hrHPV [5,6,10-13].

In a previous study, using array CGH, we identified a number of chromosomal alterations specific for hrHPVnegative HNSCCs that were absent in hrHPV-positive HNSCCs, including loss at 3p, 5q, and 9p, and amplifications at 11q [13]. The hrHPV-positive HNSCCs were characterised by a lower level of chromosomal alterations, none of which were hrHPV-specific. To investigate potential organ-independent, hrHPV-associated chromosomal alterations, genomic profiles of cervical SCCs (CxSCCs), hrHPV-positive and hrHPV-negative HNSCCs were compared using sophisticated clustering and statistical approaches $[4,13]$. In addition, we also investigated the presence of organ-specific alterations and alterations shared by all SCCs included in this study.

\section{Methods \\ CGH microarrays}

We used chromosomal profiles of $30 \mathrm{hrHPV}$-negative HNSCCs, 12 hrHPV-positive HNSCCs and 10 CxSCCs all of which were previously described (Table 1) $[4,13,14]$. To avoid possible confounding of our results, we excluded a subset of hrHPV-negative HNSCCs described in Smeets et al, which showed little to no alterations and should therefore be considered a separate group [14].

CGH BAC microarrays produced at the Microarray facility of the VU Medical Center were used. These arrays included the $1 \mathrm{Mb}$ resolution Sanger BAC clone set and a subset of clones from the Children's Hospital Oakland Research Institute (CHORI). Spots were quantified using ImaGene 5.6.1 software (BioDiscovery Ltd, Marina del Rey, CA, USA) with default settings for the flagging of bad quality spots.

The entire dataset described here is available from the Gene Expression Omnibus (GEO, http:// www.ncbi.nlm.nih.gov/projects/geo/) through series accession numbers GSE6473 (CxSCC) and GSE12020 (HNSCC).

This study followed the ethical guidelines of the Institutional Review Board of the VU University Medical Center and informed consent was obtained from all patients included.

\section{Array CGH analysis}

\section{Calling of gains and losses}

BAC clones were positioned along the genome according to the May 2004 freeze. After exclusion of clones with one or more flagged spots, the average of the triplicate spots was calculated for each BAC clone. $\log _{2}$ ratios were normalised per spotted sub-array by subtraction of the median value of all BAC clones spotted within that subarray. Segmentation and subsequent calling of gained, amplified and lost regions was done using CGHCall, an automated calling algorithm. Segments with a probability score of $\geq 0.5$ were considered gained, amplified or lost [15].

\section{Reduction of dataset into chromosomal regions}

We used the CGHregions algorithm to reduce our dataset to chromosomal regions, accepting maximally $0.1 \%$ information loss (Threshold $=0.001$ ). It was shown by Van de Wiel et al that the use of regions instead of single BAC clones improved the effectiveness of subsequent statistical analyses and facilitated interpretation of the results [16]. 
Table I: Summary of clinical data of carcinomas included in this study.

\begin{tabular}{|c|c|c|c|c|}
\hline Sample ID & origin & hrHPV (+/-) & age (yrs) & sex \\
\hline $\mathrm{CxSCC} 2$ & cervix & + & 39 & female \\
\hline CxSCC4 & cervix & + & 62 & female \\
\hline $\mathrm{CxSCCI} 2$ & cervix & + & 44 & female \\
\hline CxSCCI5 & cervix & + & 47 & female \\
\hline $\mathrm{CxSCC} 27$ & cervix & + & 49 & female \\
\hline $\mathrm{CxSCC} 28$ & cervix & + & 48 & female \\
\hline $\mathrm{CxSCC} 32$ & cervix & + & 37 & female \\
\hline $\mathrm{CxSCC} 36$ & cervix & + & 72 & female \\
\hline CxSCC38 & cervix & + & 51 & female \\
\hline CxSCC39 & cervix & + & 40 & female \\
\hline HNSCC 974 I & oropharynx & - & 53 & female \\
\hline HNSCC 9762 & oral cavity & - & 76 & female \\
\hline HNSCC 9773 & oral cavity & - & 49 & male \\
\hline HNSCC 9830 & oral cavity & - & 42 & male \\
\hline HNSCC 9848 & oropharynx & - & 59 & male \\
\hline HNSCC9892 & oral cavity & - & 67 & male \\
\hline HNSCC 9897 & oral cavity & - & 72 & male \\
\hline HNSCC 9902 & oral cavity & - & 53 & female \\
\hline HNSCC 9942 & oral cavity & - & 55 & female \\
\hline HNSCC 9952 & oral cavity & - & 61 & female \\
\hline HNSCC9956 & oral cavity & - & 38 & male \\
\hline HNSCC2OI4 & oral cavity & - & 68 & female \\
\hline HNSCC2034 & oral cavity & - & 45 & female \\
\hline HNSCC 9738 & oral cavity & - & 60 & male \\
\hline HNSCC 9745 & oropharynx & - & 52 & male \\
\hline HNSCC 9750 & oral cavity & - & 65 & male \\
\hline HNSCC 9812 & oral cavity & - & 65 & female \\
\hline HNSCC 9827 & oropharynx & - & 57 & male \\
\hline HNSCC 9829 & oral cavity & - & 74 & female \\
\hline HNSCC983I & oral cavity & - & 55 & male \\
\hline HNSCC984I & oropharynx & - & 55 & female \\
\hline HNSCC9847 & oropharynx & - & 57 & male \\
\hline HNSCC 9880 & oral cavity & - & 71 & female \\
\hline HNSCC 9907 & oral cavity & - & 46 & male \\
\hline HNSCC 9914 & oropharynx & - & 78 & male \\
\hline HNSCC 9926 & oropharynx & - & 60 & male \\
\hline HNSCC 9957 & oral cavity & - & 51 & male \\
\hline HNSCC 9970 & oral cavity & - & 49 & female \\
\hline HNSCC $998 I$ & oropharynx & - & 55 & female \\
\hline HNSCC9987 & oral cavity & - & 79 & male \\
\hline HNSCC988I & oral cavity & + & 51 & male \\
\hline HNSCC 9729 & oropharynx & + & 52 & male \\
\hline HNSCC9838 & oropharynx & + & 60 & male \\
\hline HNSCC 9860 & oropharynx & + & 67 & female \\
\hline HNSCC990I & oropharynx & + & 57 & female \\
\hline HNSCC 9948 & oropharynx & + & 70 & male \\
\hline HNSCC995I & oropharynx & + & 46 & male \\
\hline HNSCC9808 & oral cavity & + & 46 & male \\
\hline HNSCC 9859 & oral cavity & + & 40 & male \\
\hline HNSCC 9924 & oral cavity & + & 41 & female \\
\hline HNSCC 9947 & oral cavity & + & 72 & male \\
\hline HNSCC20I5 & oral cavity & + & 65 & male \\
\hline
\end{tabular}

\section{Clustering analysis}

The samples were clustered by means of a modified version of WECCA [17]. WECCA is a hierarchical clustering method tailor-made for called aCGH data. The modified version accommodates the use of call probabilities instead of calls. The use of the call probabilities in the unsupervised analysis will give a more subtle picture of the similarities and differences between the samples. The modified version of WECCA defines the distance between two features as the symmetric Kullback-Leibler divergence. The distance between the call probability profiles of two samples is then defined as the average of these divergences over all features. In the construction of the dendrogram we used Ward's linkage as it yields compact and well-separated clusters.

\section{Statistical analysis}

The association between clustering results and HPV status was determined by chi-square testing. The average total number of altered regions was compared between HPVpositive and HPV-negative carcinomas using the non-parametric Mann Whitney test. Two-sided p-values below 0.05 were considered statistically significant. Alteration patterns between HPV-positive (HNSCCs and CxSCCs) and HPV-negative tumours as well as between HNSCCs and CxSCCs were compared using a binomial differential proportion test. The test procedure includes a permutation-based false discovery rate (FDR) correction for multiple testing, needed to discriminate real differences from chance effects [18]. An FDR below 0.10 was considered statistically significant.

\section{Gene ontology analysis}

To interpret the biological significance of the genes that are located at altered chromosomal regions of interest, a gene ontology analysis was performed using Ingenuity Pathways Analysis (Ingenuity Systems ${ }^{\circledast}$, Redwood City, USA). Biological/molecular functions were considered to be significantly overrepresented if they contained more than 1 gene and the Benjamini-Hochberg corrected pvalue was $\mathrm{p}<0.10[19]$.

\section{Results hrHPV-positive carcinomas cluster together}

To obtain an overview of the similarities between samples, unsupervised hierarchical clustering was performed. This method enabled us to determine in an unbiased manner whether chromosomal profiles of hrHPV-positive HNSCCs were more closely related to hrHPV-negative HNSCCs or hrHPV-positive CxSCCs.

As is shown in Figure 1, two clusters emerged. Cluster 1 contained 24 samples, 18 of which were hrHPV-positive 


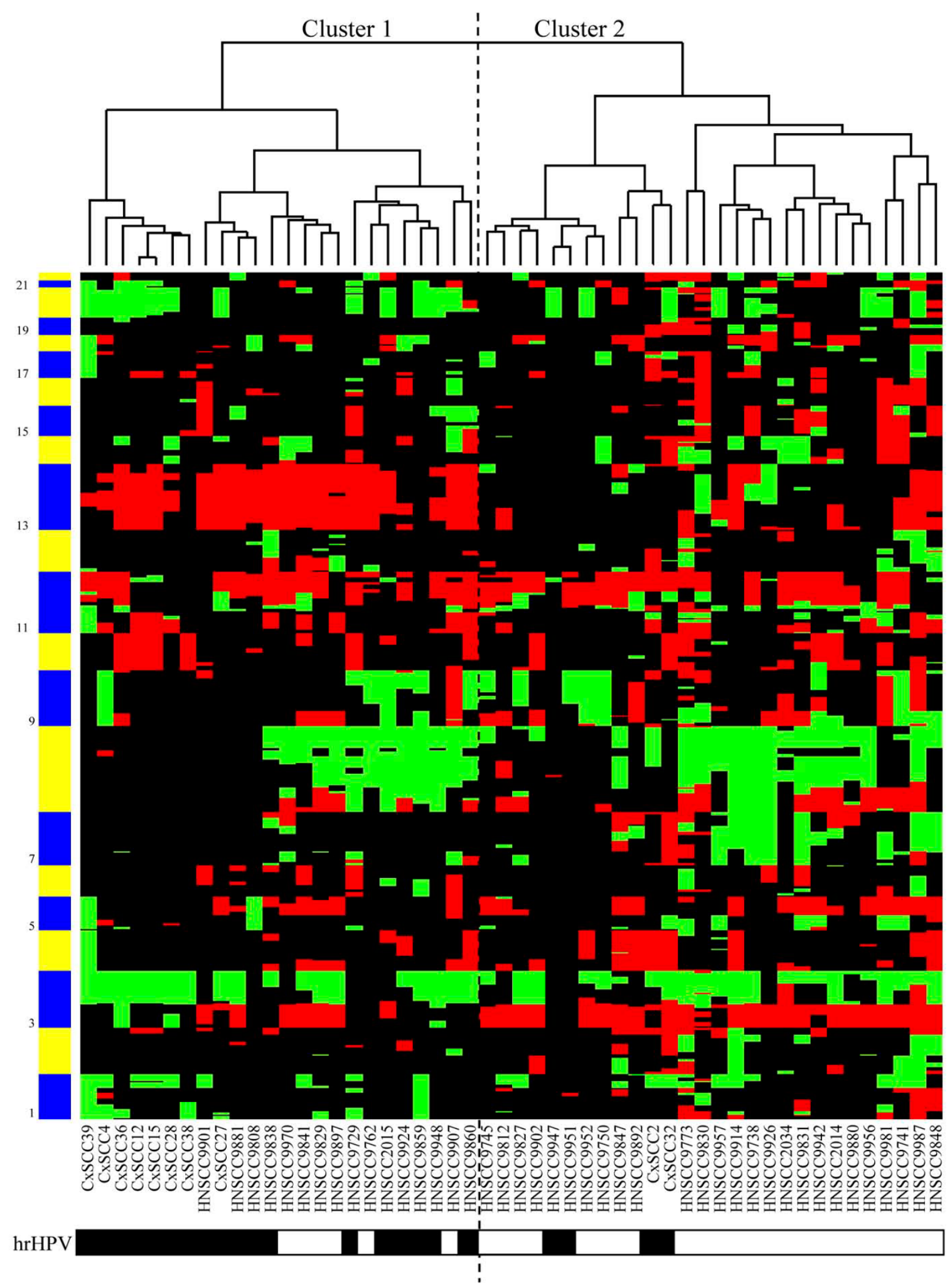

Figure I

Result from the unsupervised hierarchical clustering analysis. Cluster I contains in majority hrHPV-positive carcinomas, as is indicated by the black boxes in the legend underneath the heatmap $(p<0.000 \mathrm{I})$. 
(75\%) and 6 were hrHPV-negative. Cluster 2 contained 28 samples of which 4 samples were hrHPV-positive and 24 were hrHPV-negative (86\%). This association between cluster assignment and hrHPV status was statistically significant ( $\mathrm{p}<0.0001)$. The hrHPV-positive cluster 1 included both samples of cervical $(n=8)$ and head and neck origin $(n=10)$, indicating similarities between the chromosomal profiles of hrHPV-induced carcinomas of different anatomical origins. Within cluster 1, however, 7 out of 8 cervical samples formed a separate sub-cluster. This suggests that organ-specific alterations exist as well in hrHPV-positive HNSCCs and CxSCCs.

hrHPV-associated loss at chromosome I3q and gain at 20 q To assess the differences between all three sample groups, the frequency of gains (including amplifications) and losses was analysed for all chromosomal regions (Figure 2 ). In general, hrHPV-negative carcinomas showed significantly more altered regions than hrHPV-positive carcinomas $(\mathrm{p}=0.022)$.

To determine hrHPV-specific chromosomal alterations, the frequency of alterations was compared between hrHPV-positive (12 HNSCCs and 10 CxSCCs) and hrHPV-negative tumours (30 HNSCCs) for all chromosomal regions. Regions showing a significant difference (FDR < 0.10) in this comparison are shown in Table 2. Interestingly, loss of 13q21.1-21.33 and gain of 20p12.1q13.33 were significantly more frequent in HPV-positive SCCs compared to hrHPV-negative HNSCCs $(66.7 \%$ and $58.5 \%$ in hrHPV-positive SCCs compared to $33 \%$ and $24 \%$ in hrHPV-negative SCCs, respectively). As was also shown in our previous study, loss of regions at $3 p$ and $5 q$, and gains/amplifications of a small region at $11 \mathrm{q}$ (CCND1 locus) were specific for hrHPV-negative HNSCCs [13]. A region at 8p showed loss in hrHPV-negative HNSCCs, gain in hrHPV-positive HNSCCs and no alteration in CxSCCs. Gain at chromosome $8 \mathrm{q}$ was more frequent in hrHPV-negative HNSCCs, which was due to absence of this alteration in CxSCCs.

Our unsupervised classification results indicated that, within our hrHPV-positive cluster, CxSCCs formed a separate group. To identify potential organ-specific alterations, we therefore also compared the frequency of alterations for all regions between hrHPV-positive HNSCCs $(n=12)$ and CxSCCs $(n=10)$ (Table 3). Gains at $3 q$ and losses at $17 p$ were significantly more frequent in CxSCCs than HNSCCs (FDR < 0.10). On the other hand, HNSCCs showed frequent gains at chromosome $8 \mathrm{q}$ and losses at 11q. It is important to note that even though gain at $3 \mathrm{q}$ and loss at $11 \mathrm{q}$ were significantly different between hrHPV-positive HNSCCs and CxSCCs, these alterations were frequent in all sample groups (>50\%). Significant differences for these regions can mainly be explained by the fact that the size of the exact altered region differed between groups. Therefore, the smallest regions of overlap between all samples, namely 3q24-29 and 11q22.3-25, may represent general alterations in carcinomas derived from squamous epithelium.

All results described above are summarised in a Venn diagram, showing a general overview of the frequently altered chromosomal arms specific to or common between the (sub) groups (Figure 3).

\section{Biological functions of genes located within hrHPV-specific chromosomal alterations}

As described above gain at 20p12.1-q13.33 and loss at 13q21.1-21.33 were significantly more frequent in hrHPV-positive SCCs compared to hrHPV-negative ones. In fact, a 4.5 megabase $(\mathrm{Mb})$ region on chromosome 20 (20q11.21-q11.23), and a $2 \mathrm{Mb}$ region on chromosome 13 (13q21.1) formed the smallest regions of overlap (SRO) at these respective loci when all hrHPV-positive carcinomas were considered (Figure $4 \mathrm{~A}$ and $4 \mathrm{~B}$ ). The SRO at chromosome $20 \mathrm{q}$ contains 78 genes and the one at chromosome 13q contains 6 genes [see Additional file 1]. Within the SRO at chromosome 20, two genes reside, i.e. NCOA6 and RBM39, which showed elevated expression in hrHPV16 E7 expressing cells in vitro [20]. Other cancerrelated genes located within this SRO include PIGU, E2F1, and DNMT3B. The SRO on chromosome 13 encompasses the PCDH17 gene and a cluster of five identical loci all of which are predicted to encode proline-rich proteins that contain several dopamine D4 receptor signatures.

Subsequent gene ontology analysis of all genes located in these SROs identified a number of significantly overrepresented GO biological/molecular functions, including cell cycle, cell-to-cell signalling and interaction, cellular growth and proliferation, and a number of cellular maintenance functions (i.e. DNA replication, recombination and repair, gene expression, cellular function and maintenance, cellular assembly and organisation, cellular compromise, cell death, cell morphology, cellular development, nucleic acid metabolism, and lipid metabolism).

\section{Discussion}

In the present study we compared genome-wide chromosomal profiles of hrHPV-negative HNSCCs, hrHPV-positive HNSCCs and hrHPV-positive CxSCCs, to determine whether hrHPV-associated carcinomas of different origins have similar chromosomal signatures. In addition, potential organ-specific alterations were determined within the total group of hrHPV-positive SCCs.

Unsupervised hierarchical clustering resulted in a separate hrHPV-positive cluster, indicating similarities in the chro- 

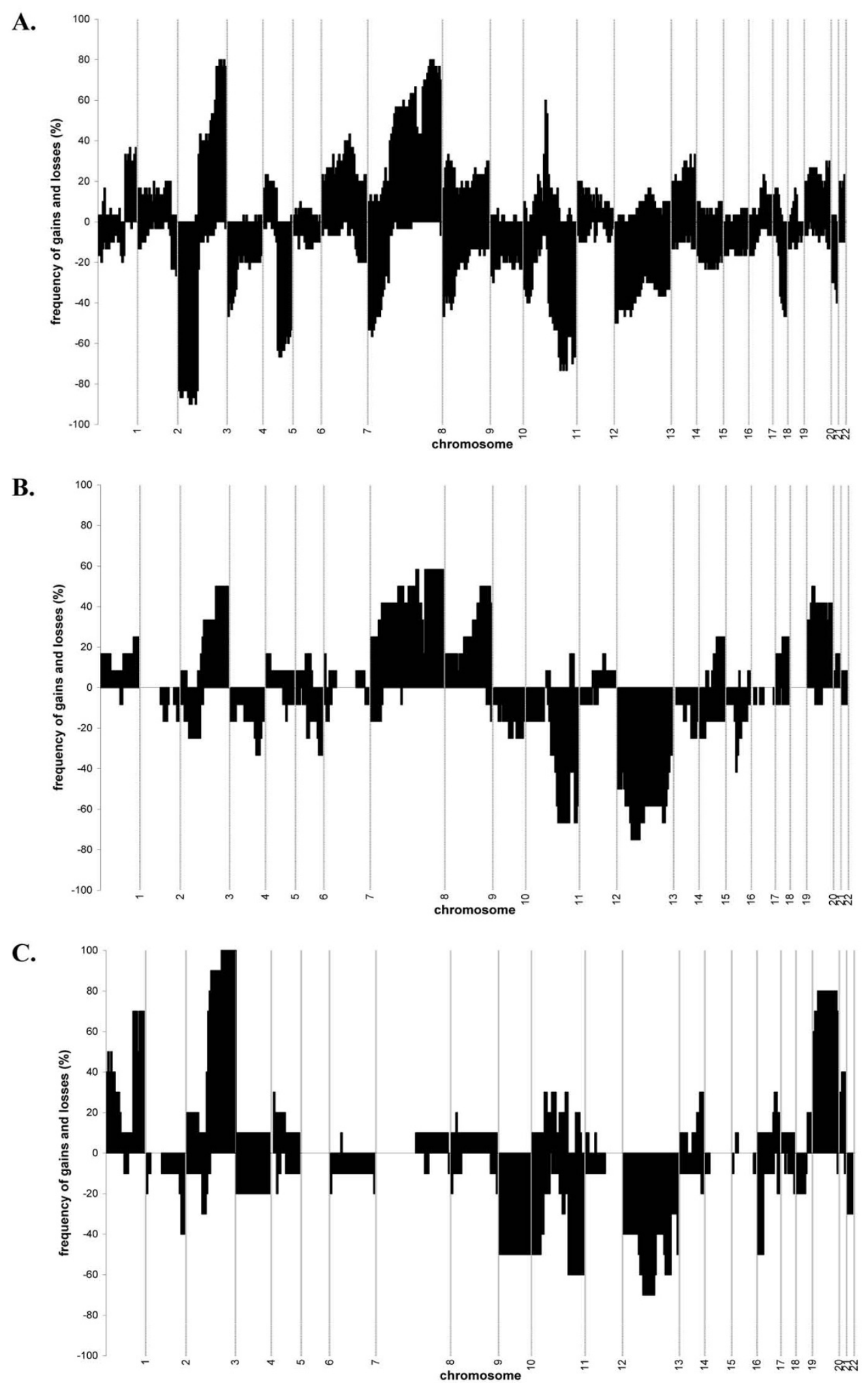

Figure 2

Frequency plots for all 3 sample groups. The frequency of gains (positive axis) and losses (negative axis) are shown for $\mathbf{A}$. hrHPV-negative HNSCCs, B. hrHPV-positive HNSCCs and C. CxSCCs for chromosome I-22. 
Table 2: Significantly different chromosomal alterations between hrHPV+ and hrHPV- carcinomas.

\begin{tabular}{|c|c|c|c|c|c|c|c|c|c|c|}
\hline \multirow[b]{2}{*}{ chromosome } & \multirow[b]{2}{*}{ Start (Mb) } & \multirow[b]{2}{*}{ End (Mb) } & \multirow[b]{2}{*}{ Cytoband } & \multirow[b]{2}{*}{ FDR } & \multicolumn{3}{|c|}{ HPV+ (HNSCC; CxSCC) } & \multicolumn{3}{|c|}{ HPV- } \\
\hline & & & & & $\%$ loss & $\%$ gain & $\%$ amp & $\%$ loss & \%gain & $\%$ amp \\
\hline 1 & 1.08 & 58.69 & $1 \mathrm{p} 36.33-\mathrm{p} 32.2$ & 0.08 & 0.0 & $\begin{array}{c}26.7 \\
(15.6 ; 40.0)\end{array}$ & 0.0 & 14.6 & 5.8 & 0.0 \\
\hline 1 & 109.78 & 117.23 & |p|3.3-p|3.I & 0.04 & 0.0 & $\begin{array}{c}13.6 \\
(16.7 ; 10.0)\end{array}$ & 0.0 & 17.8 & 0.0 & 0.0 \\
\hline 2 & 96.17 & 97.68 & $2 q 11.2$ & 0.08 & $4.5(0.0 ; 10.1)$ & 0.0 & 0.0 & 0.0 & 20.0 & 0.0 \\
\hline 2 & 165.89 & 180.53 & $2 q 24.3-q 31.3$ & 0.06 & $9.1(8.3 ; 10.0)$ & 0.0 & 0.0 & 0.0 & 18.9 & 0.0 \\
\hline 3 & 0.28 & 86.18 & $3 p 26.3-p / 2.1$ & 0.00 & $\begin{array}{c}17.8 \\
(20.5 ; / 4.6)\end{array}$ & $9.4(3.8 ; 16.2)$ & 0.0 & 86.2 & 0.5 & 0.0 \\
\hline 5 & 51.16 & 180.57 & $5 q 11.2-q 35.3$ & 0.00 & $8.6(7.5 ; 10.0)$ & $9.1(8.3 ; 10.0)$ & 0.0 & 61.0 & 0.3 & 0.0 \\
\hline 7 & 9.95 & 25.67 & 7p21.3-p15.2 & 0.05 & $\begin{array}{c}13.6 \\
(16.7 ; 10.0)\end{array}$ & 0.0 & 0.0 & 3.3 & 21.7 & 0.0 \\
\hline 7 & 65.52 & 116.45 & $7 q 11.21-q 31.2$ & 0.03 & $4.5(0.0 ; 10.0)$ & $0.3(0.6 ; 0.0)$ & 0.0 & 4.1 & 32.1 & 2.8 \\
\hline 8 & 0.38 & 34.51 & 8p23.3-pl2 & 0.02 & $6.8(12.5 ; 0.0)$ & $17.3(31.7 ; 0.0)$ & 0.0 & 48.7 & 12.0 & 0.0 \\
\hline 8 & 75.65 & 93.93 & $8 q 21.11-q 22.1$ & 0.06 & 0.0 & $22.7(41.7 ; 0.0)$ & 0.0 & 3.3 & 57.8 & 0.0 \\
\hline 8 & 114.72 & 145.68 & $8 q 23.3-q 24.3$ & 0.05 & $0.3(0.0 ; 0.7)$ & $34.4(55.4 ; 9.3)$ & 0.0 & 0.5 & 70.2 & 2.1 \\
\hline 11 & 69.18 & 70.31 & $1 / q / 3.3-q / 3.4$ & 0.04 & $\begin{array}{c}11.4 \\
(12.5 ; 10.0)\end{array}$ & $13.6(0.0 ; 30.0)$ & $4.5(8.3 ; 0.0)$ & 10.0 & 15.0 & 41.7 \\
\hline 13 & 55.72 & 71.49 & $\begin{array}{l}13 q 21.1- \\
\text { q21.33 }\end{array}$ & 0.06 & $\begin{array}{c}66.7 \\
(64.8 ; 68.9)\end{array}$ & 0.0 & 0.0 & 33.0 & 7.0 & 2.2 \\
\hline 14 & 47.08 & 61.97 & $|4 q 2| .3-q 23.2$ & 0.08 & $\begin{array}{c}11.4 \\
(12.5 ; 10.0)\end{array}$ & $2.3(0.0 ; 5.0)$ & 0.0 & 8.3 & 28.3 & 0.0 \\
\hline 14 & 79.43 & 86.91 & $|4 q 31.1-q 3| .3$ & 0.09 & $\begin{array}{c}18.2 \\
(25.0 ; 10.0)\end{array}$ & $9.1(0.0 ; 20.0)$ & 0.0 & 6.7 & 33.3 & 0.0 \\
\hline 18 & 28.34 & 75.62 & $18 q 12.1-q 23$ & 0.03 & $10.0(8.3 ; 12.0)$ & $\begin{array}{c}18.2 \\
(25.0 ; 10.0)\end{array}$ & 0.0 & 42.7 & 2.0 & 0.0 \\
\hline 20 & 15.56 & 60.28 & $\begin{array}{l}20 p / 2.1- \\
q \mid 3.33\end{array}$ & 0.06 & I.5 $(2.8 ; 0.0)$ & $\begin{array}{c}58.5 \\
(41.7 ; 78.7)\end{array}$ & 0.0 & 6.2 & 24.0 & 0.0 \\
\hline 21 & 14.68 & 46.85 & $21 q 11.2-q 22.3$ & 0.00 & I.I (0.0; 2.5) & $\begin{array}{c}25.0 \\
(14.6 ; 37.5)\end{array}$ & 0.0 & 33.3 & 2.5 & 0.0 \\
\hline
\end{tabular}

hrHPV-specific alterations are printed in bold. Alterations specific for hrHPV-negative HNSCCs are italicised. Mb; Megabases, FDR; False Discovery Rate, amp; amplification

mosomal profiles of hrHPV-induced carcinomas. Subsequent supervised statistical analysis identified a number of hrHPV-associated chromosomal alterations, including gains at 20p13-q13.33 and losses at 13q21.1-21.33, which were frequent $(>50 \%)$ in hrHPV-positive carcinomas, but were only rarely observed in hrHPV-negative carcinomas. In contrast, lost regions at $3 p$ and $5 q$ as well as amplifications at 11q13.3 (CCND1 locus) were frequent in hrHPV-negative carcinomas, but not in hrHPV-positive carcinomas. In our previous study comparing only hrHPV-positive and hrHPV-negative HNSCCs, the same regions were identified as well as being specific for hrHPVnegative HNSCCs [13].

In the present study we found that gains at $20 \mathrm{q}$ and losses at $13 \mathrm{q}$ are specific for hrHPV-positive carcinomas of dif-

Table 3: Significantly different chromosomal alterations between hrHPV-positive HNSCCs and CxSCCs.

\begin{tabular}{|c|c|c|c|c|c|c|c|c|c|c|}
\hline \multirow[b]{2}{*}{ chromosome } & \multirow[b]{2}{*}{ start (Mb) } & \multirow[b]{2}{*}{ end (Mb) } & \multirow[b]{2}{*}{ Cytoband } & \multirow[b]{2}{*}{ FDR } & \multicolumn{3}{|c|}{ HNSCC+ } & \multicolumn{3}{|c|}{ CxSCC } \\
\hline & & & & & $\%$ loss & \% gain & $\%$ amp & $\%$ loss & \%gain & $\%$ amp \\
\hline 3 & 112.84 & 199.07 & $3 q 13.2-q 29$ & 0.09 & 0.0 & 40.6 & 2.1 & 0.0 & 95.6 & 0.0 \\
\hline 8 & 61.67 & 74.46 & $8 q \mid 2.1-q 2 I .1 I$ & 0.09 & 2.1 & 50.0 & 0.0 & 0.0 & 0.0 & 0.0 \\
\hline 8 & 94.15 & 99.29 & $8 q 22.1-q 22.2$ & 0.09 & 0.0 & 50.0 & 0.0 & 0.0 & 0.0 & 0.0 \\
\hline 8 & | 42.29 & 145.68 & $8 q 24.3$ & 0.09 & 0.0 & 58.3 & 0.0 & 10.0 & 0.0 & 0.0 \\
\hline II & 98.40 & 105.02 & I Iq22.I-q22.3 & 0.09 & 66.7 & 0.0 & 0.0 & 20.0 & 30.0 & 0.0 \\
\hline 17 & 0.91 & 2.40 & $17 p \mid 3.3$ & 0.09 & 0.0 & 0.0 & 0.0 & 50.0 & 10.0 & 0.0 \\
\hline 17 & 19.19 & 19.29 & $|7 p| \mid .2$ & 0.09 & 0.0 & 0.0 & 0.0 & 50.0 & 10.0 & 0.0 \\
\hline
\end{tabular}

Alterations specific for HNSCCs are printed in bold, alterations specific for CxSCCs are italicised. Mb;Megabases, FDR; False Discovery Rate, amp; amplification 


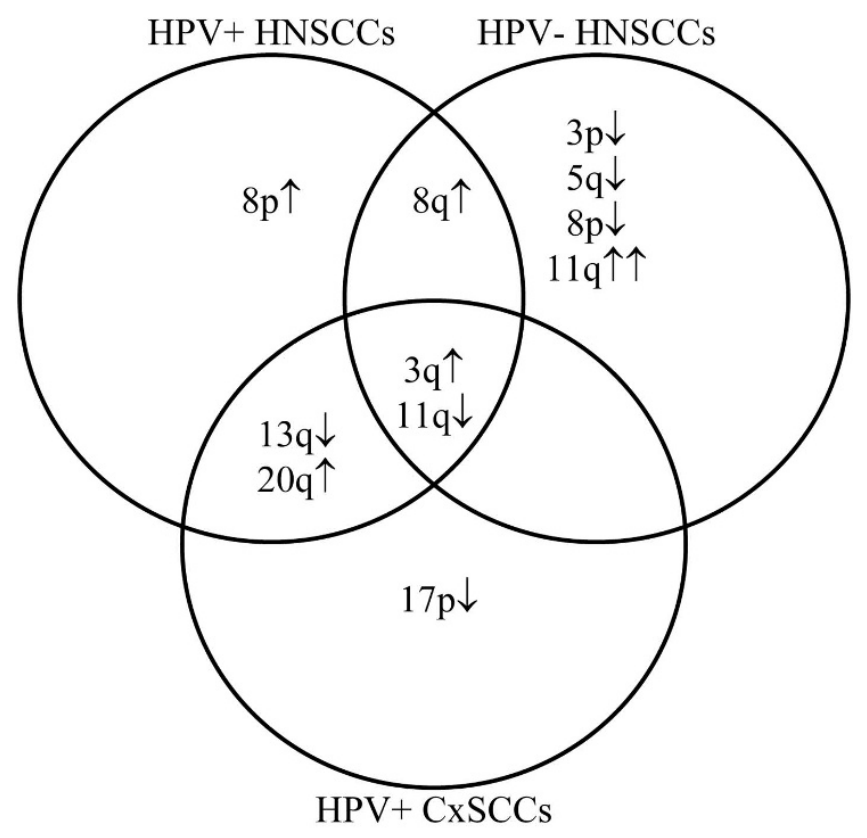

Figure 3

Summary of common and specific chromosomal alterations in the different sample groups. Chromosome arms showing frequent alterations (>50\%) in one or more sample groups (hrHPV-positive HNSCCs; hrHPV-negative HNSCCs; CxSCCs) are shown in a Venn diagram. $\uparrow$ indicates gain; $\downarrow$ indicates loss

ferent anatomical origins. Our previous study, including only hrHPV-positive and hrHPV-negative HNSCCs, did not identify these alterations as specific for hrHPV-positive HNSCCs. However, in the present study a larger number of hrHPV-negative hrHNSCCs and HPV-positive SCCs was included. Furthermore, a sophisticated, objective calling method was presently used to determine gained and lost regions [15]. Interestingly, in a number of studies describing hrHPV E6 and/or E7 mediated immortalisation of keratinocytes of multiple anatomical origins, gains of chromosome $20 \mathrm{q}$ and losses of chromosome $13 \mathrm{q}$ were consistently observed [20-23]. Low-level gains of chromosome $20 \mathrm{q}$ are suggested to be caused by E7 expression and consequent inactivation of the $\mathrm{pRb}$ pathway in epithelial cells $[20,21,24]$. Deletion of part of the long arm of chromosome 13 is frequently found in a number of hrHPV-associated tumours, including cervical, anal and head and neck carcinomas $[4,13,25]$. Sabbir et al showed that loss of $13 \mathrm{q}$ in HNSCCs was associated with the presence of hrHPV, which is in agreement with our findings [26].

In a recent study Pyeon et al showed that the gene expression patterns of hrHPV-positive HNSCCs and CxSCCs dif- fered yet shared many changes compared to hrHPVnegative HNSCCs [10]. Our study shows that the same holds true on a chromosomal level. Interestingly, $28 \%$ of the genes Pyeon et al found to be differentially expressed between hrHPV-positive and hrHPV-negative carcinomas is located within the chromosomal regions identified in this study and showed expression changes concordant with the chromosomal alterations. Of these genes, 39\% was located at chromosome $1 p, 25 \%$ at $5 q$, and $14 \%$ at $3 p$. The other genes were located at $11 \mathrm{q}, 18 \mathrm{q}$ and $21 \mathrm{q}$. Only one gene, SYCP2, was located within the hrHPV-specific chromosomal alterations found in this study (20q), but was not located within our SRO. To the best of our knowledge none of the genes are known to directly interact with hrHPVE6 and/or E7 $[27,28]$. Pathway analysis of all genes overlapping with our findings identified cell cycle/proliferation as most overrepresented biological function, which is in concordance with the observations made by Pyeon et al [10].

Pathway analysis of all genes located within the hrHPVassociated SROs at chromosome $20 \mathrm{q}$ and $13 \mathrm{q}$ found in this study, again underlined the importance of cell cycle (replication and proliferation) related genes in hrHPVmediated carcinogenesis. This may be related to the continuous E7-regulated E2F1 activation and is accompanied by changes in overall cellular maintenance systems, such as nucleic acid metabolism, as was also found in HPV16 E7 expressing epithelial cells in vitro [20]. Two genes, NCOA6 and RBM39, overlapped between this in vitro study and our results, warranting further investigation of their role in hrHPV-mediated transformation. NCOA6 encodes a transcriptional coactivator interacting with basal transcription factors, histone acetyltransferases, and methyltransferases. RBM39 encodes an RNA binding protein and possible splicing factor and may act as a transcriptional coactivator for the AP-1 transcription activator complex and estrogen receptors. Other cancer-related genes at 20q include E2F1, which is specifically targeted by hrHPV-mediated degradation of pRb, PIGU, which may play a role in cell cycle control and was identified as an oncogene in bladder cancer [29], and DNMT3B, a de novo DNA methyl transferase. We previously showed that DNMT3B is amplified in the cervical cancer cell line $\mathrm{SiHa}$ and found a correlation between increased DNMT3B gene copy numbers and elevated mRNA expression in $78 \%$ of CxSCCs [4]. The increased DNMT3B levels are most likely related to the high frequency of tumour suppressor gene promoter hypermethylation events during hrHPV-mediated carcinogenesis [30-32]. The SRO at chromosome 13q includes one known gene, PCDH17, a member of the protocadherin family, which is a subfamily of the cadherin superfamily. At present no reports are available describing interactions between hrHPV and protocadherins. How- 
Figure 4

Losses on chromosome 13 in hrHPV+ tumours

A.

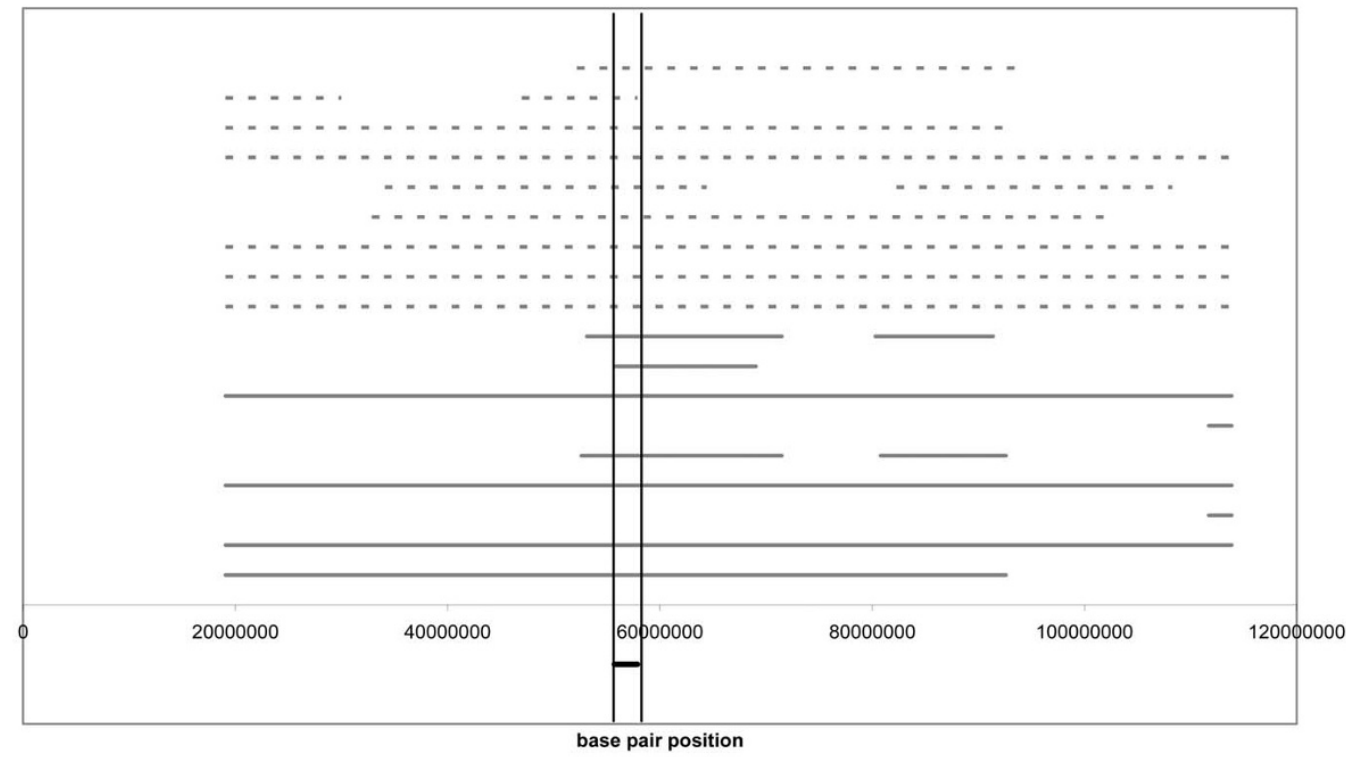

Gains on chromosome 20 in hrHPV+ tumours

B.

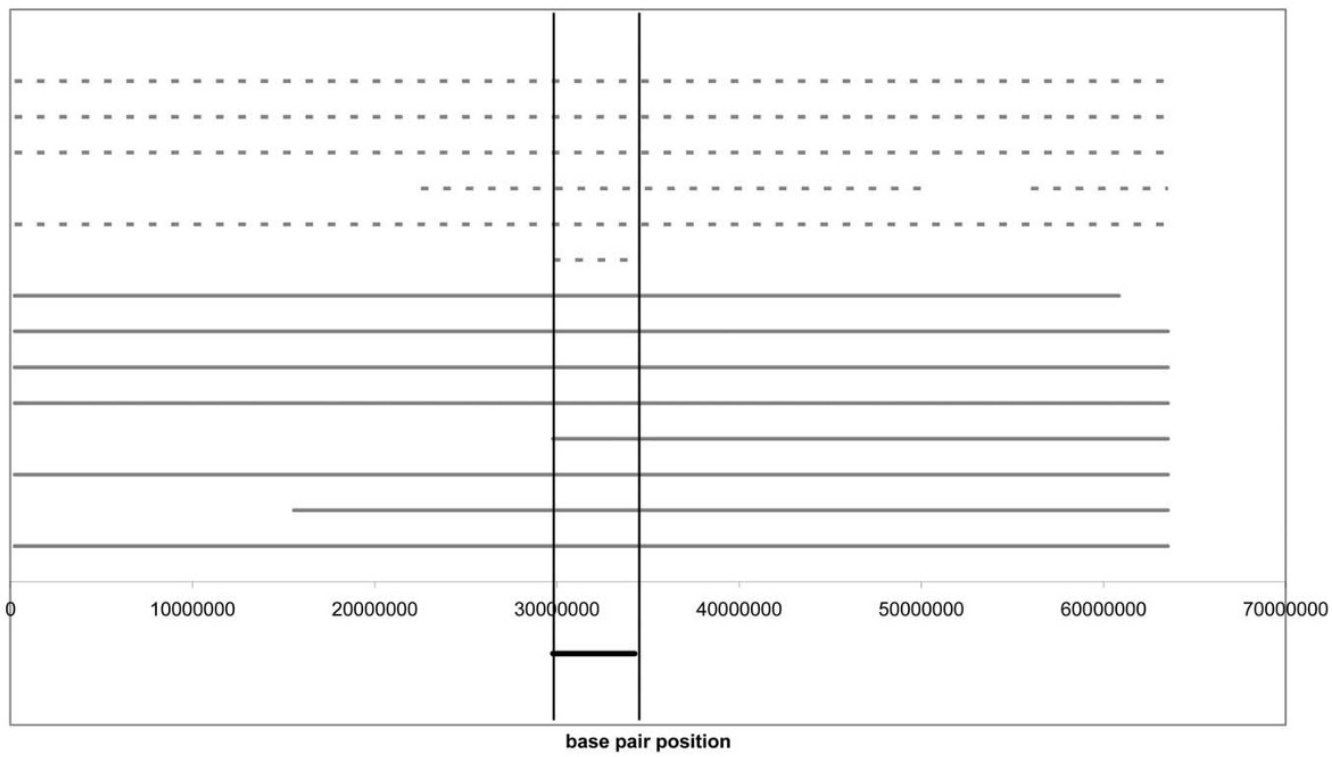

C.

\begin{tabular}{ccccc} 
chromosome & start position (bp) & end position (bp) & cytoband & number of tumours \\
\hline \hline 13 & 55718989 & 57862597.5 & $13 q 21.1$ & $16(72.7 \%)$ \\
20 & 29779091.5 & 34257710.5 & $20 q 11.21-q 11.23$ & $14(63.4 \%)$
\end{tabular}

Figure 4

Genomic coordinates of A. losses at chromosome I 3 and B. gains at chromosome 20 are shown for all hrHPVpositive carcinomas. Chromosomal alterations in CxSCCs are shown by dashed lines and alterations in hrHPV-positive HNSCCs by solid lines. In C. the smallest regions of overlap (SROs) between hrHPV-positive carcinomas at chromosome I3 and 20 are summarised. 
ever hrHPV presence has been related to decreased E-cadherin and subsequent impaired immune response [3335].

Next to the hrHPV-related common events, organ-specific alterations for CxSCCs and HNSCCs were identified as well. CxSCCs more frequently showed loss at $17 \mathrm{p}$, while HNSCCs were characterised by frequent gains at chromosome 8q, harbouring the oncogene c-Myc [36].

Gains at $3 \mathrm{q}$ and losses at $11 \mathrm{q}$ were found frequently in all SCCs included in this study, suggesting their involvement in carcinogenesis of squamous epithelial cells in general. Interestingly, we previously showed that gains of $3 \mathrm{q}$ were highly frequent in CxSCCs but not in adenocarcinomas of the same organ, further emphasising that this alteration may be specific for squamous epithelium [4].

\section{Conclusion}

Together with results from previous studies, our findings support a causal role for hrHPV in the development of a subset of HNSCCs. Consequently, hrHPV-positive and hrHPV-negative HNSCCs should be regarded as different disease entities requiring different diagnostic and therapeutic approaches. The fact that hrHPV-associated SCCs of different organs have chromosomal alterations in common, suggests that these alterations are crucial for hrHPVinduced carcinogenesis. Diagnostic and/or therapeutic targets based on these alterations may therefore be relevant to hrHPV-associated SCCs of all anatomical origins.

\section{Abbreviations}

hrHPV: high-risk human papillomavirus; CxSCC: squamous cell carcinoma of the uterine cervix; HNSCC: squamous cell carcinoma of the head and neck; SCC: squamous cell carcinoma; CGH: comparative genomic hybridisation; BAC: bacterial artificial chromosome; $\mathrm{Mb}$ : megabase; FDR: false discovery rate; SRO: smallest region of overlap; GO: gene ontology.

\section{Competing interests}

The authors declare that they have no competing interests.

\section{Authors' contributions}

SMW and SJS performed array experiments and data analysis and drafted the manuscript. RDMS and BJMB participated in the design of the study and the drafting of the manuscript. WNvW and MAvdW contributed to the statistical analyses. BY and GAM were involved in the development of the micorarray platform and provided the facilities for the microarray experiments. PJFS, CRL, CJLMM and RHB contributed to the conception of the study and critically revised the manuscript. All authors read and approved the final manuscript.

\section{Additional material}

\section{Additional file 1}

BAC clones and genes included in the SROs of the hrHPV-specific chromosomal alterations at chromosomes $13 q$ and $20 q$. All BAC clones and genes located within the identified SROs at chromosome 20q and $13 q$ are listed here.

Click here for file

[http://www.biomedcentral.com/content/supplementary/17558794-2-32-S1.doc]

\section{Acknowledgements}

We would like to thank the Mapping Core and Map Finishing groups of the Wellcome Trust Sanger Institute for initial clone supply and verification. This work was partly supported by the Centre for Medical Systems Biology (CMSB) in the framework of the Netherlands Genomic Initiative (NGI).

\section{References}

I. zur Hausen H: Papillomaviruses and cancer: from basic studies to clinical application. Nat Rev Cancer 2002, 2:342-350.

2. Steenbergen RD, de Wilde J, Wilting SM, Brink AA, Snijders PJ, Meijer CJ: HPV-mediated transformation of the anogenital tract. J Clin Virol 2005, 32(SuppI I):S25-S33.

3. zur Hausen $\mathrm{H}$ : Papillomaviruses causing cancer: evasion from host-cell control in early events in carcinogenesis. J Natl Cancer Inst 2000, 92:690-698.

4. Wilting SM, Snijders PJ, Meijer GA, Ylstra B, ljssel PR van den, Snijders AM, Albertson DG, Coffa J, Schouten JP, Wiel MA van de, et al: Increased gene copy numbers at chromosome 20q are frequent in both squamous cell carcinomas and adenocarcinomas of the cervix. J Pathol 2006, 209:220-230.

5. Braakhuis BJ, Snijders PJ, Keune WJ, Meijer CJ, Ruijter-Schippers HJ, Leemans CR, Brakenhoff RH: Genetic patterns in head and neck cancers that contain or lack transcriptionally active human papillomavirus. J Natl Cancer Inst 2004, 96:998-1006.

6. Dahlgren L, Mellin H, Wangsa D, Heselmeyer-Haddad K, Bjornestal L, Lindholm J, Munck-Wikland E, Auer G, Ried T, Dalianis T: Comparative genomic hybridization analysis of tonsillar cancer reveals a different pattern of genomic imbalances in human papillomavirus-positive and -negative tumors. Int J Cancer 2003, 107:244-249.

7. Gillison ML, Koch WM, Capone RB, Spafford M, Westra WH, Wu L, Zahurak ML, Daniel RW, Viglione M, Symer DE, et al.: Evidence for a causal association between human papillomavirus and a subset of head and neck cancers. J Natl Cancer Inst 2000, 92:709-720.

8. McKaig RG, Baric RS, Olshan AF: Human papillomavirus and head and neck cancer: epidemiology and molecular biology. Head Neck 1998, 20:250-265.

9. Hoffmann M, Gorogh T, Gottschlich S, Lohrey C, Rittgen W, Ambrosch P, Schwarz E, Kahn T: Human papillomaviruses in head and neck cancer: 8 year-survival-analysis of 73 patients. Cancer Lett 2005, 21 8: 199-206.

10. Pyeon D, Newton MA, Lambert PF, den Boon JA, Sengupta S, Marsit C), Woodworth CD, Connor JP, Haugen TH, Smith EM, et al:: Fundamental differences in cell cycle deregulation in human papillomavirus-positive and human papillomavirus-negative head/neck and cervical cancers. Cancer Res 2007, 67:4605-46I9.

II. Schlecht NF, Burk RD, Adrien L, Dunne A, Kawachi N, Sarta C, Chen Q, Brandwein-Gensler M, Prystowsky MB, Childs G, et al:: Gene expression profiles in HPV-infected head and neck cancer. J Pathol 2007, 21 3:283-293.

12. Slebos RJ, Yi Y, Ely K, Carter J, Evjen A, Zhang X, Shyr Y, Murphy BM, Cmelak AJ, Burkey BB, et al:: Gene expression differences associated with human papillomavirus status in head and neck squamous cell carcinoma. Clin Cancer Res 2006, I 2:70 I-709.

13. Smeets SJ, Braakhuis BJ, Abbas S, Snijders PJ, Ylstra B, Wiel MA van de, Meijer GA, Leemans CR, Brakenhoff RH: Genome-wide DNA 
copy number alterations in head and neck squamous cell carcinomas with or without oncogene-expressing human papillomavirus. Oncogene 2006, 25:2558-2564.

14. Smeets SJ, Brakenhoff RH, Ylstra B, van Wieringen WN, Wiel MA van de, Leemans CR, Braakhuis BJ: Genetic classification of oral and oropharyngeal carcinomas identifies subgroups with a different prognosis. Cell Oncol 2009 in press.

15. Wiel MA van de, Kim KI, Vosse SJ, van Wieringen WN, Wilting SM, Ylstra B: CGHcall: calling aberrations for array CGH tumor profiles. Bioinformatics 2007, 23:892-894.

16. Wiel MA van de, van Wieringen WN: CGHRegions: Dimension Reduction for Array CGH Data with Minimal Information Loss. Cancer Informatics 2007, 2:55-63.

17. van Wieringen WN, Wiel MA van de, Ylstra B: Weighted clustering of called array CGH data. Biostatistics 2008, 9:484-500.

18. Manduchi E, Grant GR, McKenzie SE, Overton GC, Surrey S, Stoeckert CJ Jr: Generation of patterns from gene expression data by assigning confidence to differentially expressed genes. Bioinformatics 2000, 16:685-698.

19. Benjamini Y, Drai D, Elmer G, Kafkafi N, Golani I: Controlling the false discovery rate in behavior genetics research. Behav Brain Res 200I, I 25:279-284.

20. Klingelhutz AJ, Qian Q, Phillips SL, Gourronc FA, Darbro BW, Patil $S R$ : Amplification of the chromosome $20 q$ region is associated with expression of HPV- 16 E7 in human airway and anogenital epithelial cells. Virology 2005, 340:237-244.

21. Jin Y, Zhang H, Tsao SW, Jin C, Lv M, Strombeck B, Wiegant J, Wan TS, Yuen PW, Kwong YL: Cytogenetic and molecular genetic characterization of immortalized human ovarian surface epithelial cell lines: consistent loss of chromosome 13 and amplification of chromosome 20. Gynecol Oncol 2004, 92: $|83-19|$

22. Savelieva E, Belair CD, Newton MA, DeVries S, Gray JW, Waldman F, Reznikoff CA: 20q gain associates with immortalization: 20 q 3.2 amplification correlates with genome instability in human papillomavirus 16 E7 transformed human uroepithelial cells. Oncogene 1997, |4:55|-560.

23. Steenbergen RD, Hermsen MA, Walboomers JM, Meijer GA, Baak JP, Meijer CJ, Snijders PJ: Non-random allelic losses at 3p, I I p and I3q during HPV-mediated immortalization and concomitant loss of terminal differentiation of human keratinocytes. Int J Cancer 1998, 76:4I2-4I7.

24. Cuthill S, Agarwal P, Sarkar S, Savelieva E, Reznikoff CA: Dominant genetic alterations in immortalization: role for $20 \mathrm{q}$ gain. Genes Chromosomes Cancer 1999, 26:304-31 I.

25. Heselmeyer K, du MS, Blegen H, Friberg B, Svensson C, Schrock E, Veldman T, Shah K, Auer G, Ried T: A recurrent pattern of chromosomal aberrations and immunophenotypic appearance defines anal squamous cell carcinomas. $\mathrm{Br} J$ Cancer 1997, 76: $127 \mid-1278$.

26. Sabbir MG, Roy A, Mandal S, Dam A, Roychoudhury S, Panda CK: Deletion mapping of chromosome I3q in head and neck squamous cell carcinoma in Indian patients: correlation with prognosis of the tumour. Int J Exp Pathol 2006, 87:I5I-I6I.

27. Laughlin-Drubin ME, Munger K: The human papillomavirus E7 oncoprotein. Virology 2009, 384:335-344.

28. Wise-Draper TM, Wells SI: Papillomavirus E6 and E7 proteins and their cellular targets. Front Biosci 2008, I3:1003-1017.

29. Guo Z, Linn JF, Wu G, Anzick SL, Eisenberger CF, Halachmi S, Cohen Y, Fomenkov A, Hoque MO, Okami K, et al.: CDC9ILI (PIG-U) is a newly discovered oncogene in human bladder cancer. Nat Med 2004, 10:374-38I.

30. Henken FE, Wilting SM, Overmeer RM, van Rietschoten JG, Nygren AO, Errami A, Schouten JP, Meijer CJ, Snijders PJ, Steenbergen RD: Sequential gene promoter methylation during HPV-induced cervical carcinogenesis. Br J Cancer 2007, 97:|457-|464.

31. Wentzensen N, Sherman ME, Schiffman M, Wang SS: Utility of methylation markers in cervical cancer early detection: appraisal of the state-of-the-science. Gynecol Oncol 2009, I I 2:293-299.

32. Whiteside MA, Siegel EM, Unger ER: Human papillomavirus and molecular considerations for cancer risk. Cancer 2008, I | 3:298|-2994.

33. Al Moustafa AE, Kassab A, Darnel A, Yasmeen A: High-risk HPVI ErbB-2 interaction on $E$-cadherin/catenin regulation in human carcinogenesis. Curr Pharm Des 2008, I 4:2159-2I 72.
34. Caberg JH, Hubert PM, Begon DY, Herfs MF, Roncarati PJ, Boniver J], Delvenne PO: Silencing of E7 oncogene restores functional Ecadherin expression in human papillomavirus 16-transformed keratinocytes. Carcinogenesis 2008, 29:|44|-|447.

35. Hubert P, Caberg JH, Gilles C, Bousarghin L, Franzen-Detrooz E, Boniver J, Delvenne P: E-cadherin-dependent adhesion of dendritic and Langerhans cells to keratinocytes is defective in cervical human papillomavirus-associated (pre)neoplastic lesions. J Pathol 2005, 206:346-355.

36. Issing WJ, Wustrow TP, Heppt WJ: Oncogenes related to head and neck cancer. Anticancer Res 1993, I3:254I-255I.

\section{Pre-publication history}

The pre-publication history for this paper can be accessed here:

http://www.biomedcentral.com/1755-8794/2/32/prepub

Publish with Bio Med Central and every scientist can read your work free of charge

"BioMed Central will be the most significant development for disseminating the results of biomedical research in our lifetime. "

Sir Paul Nurse, Cancer Research UK

Your research papers will be:

- available free of charge to the entire biomedical community

- peer reviewed and published immediately upon acceptance

- cited in PubMed and archived on PubMed Central

- yours - you keep the copyright

Submit your manuscript here:

http://www.biomedcentral.com/info/publishing_adv.asp
BiolMedcentral 